\title{
Effects of a synbiotic on intestinal and immune functions of healthy adults
}

\author{
B. Viadel ${ }^{1}$, E. Nova ${ }^{2}$ J. E. Carreres ${ }^{1}$ and A. $\operatorname{Marcos}^{2}$ \\ ${ }^{1}$ Department of New Products, AINIA, Technologic Center, Valencia, Spain and ${ }^{2}$ Immunonutrition Group, \\ Department of Metabolism and Nutrition, Instituto del Frío, CSIC, Madrid, Spain
}

Synbiotic preparations contain a combination of probiotics and prebiotics (fermentable fibre). The principal purported health-promoting effect of probiotics is the enhancement of mucosal immune defences, while fructose oligosaccharides have a bifidogenic effect on human colonic endogenous flora. The main objective of the present study was to evaluate the effects of a 6-week intervention with a synbiotic product (Lactobacillus acidophilus, Lactobacillus delbrueckii ssp. bulgaricus, Lactobacillus paracasei ssp. paracasei, Streptococcus thermophilus, Bifidobacterium sp. $\left(2.4 \times 10^{9}\right.$ colony-forming units/d) and fructo-oligosaccharides; SYMBYO ${ }^{\circledR}$ : Laboratorios Phergal SA, Madrid, Spain) on the intestinal and immune functions of thirty-six healthy adults aged 25-45 years (sixteen males, twenty females). Subjects were randomly assigned to the symbiotic ( $\mathrm{S}$; seven males, eleven females) or placebo (C; nine males, nine females) groups. Faecal and blood samples were collected at baseline and on completion of the study. Total aerobic and anaerobic micro-organisms, enterobacteria, enterococci, pseudomonas, yeasts, lactobacilli, bacteroids, Clostridium spp. and bifidobacteria were quantified in faeces. Phagocyte function was quantitatively assessed (Phagotest; BD Biosciences, Madrid, Spain) and highly-sensitive C-reactive protein, caeruloplasmin (immunoturbidimetry) and soluble adhesion molecules intercellular adhesion molecule-1 (sICAM-1), vascular cell adhesion molecule-1 (sVCAM-1) and sL-selectin (ELISA) were determined in serum. Intestinal motility and improvement in any previous gastrointestinal symptoms were assessed through a self-administered questionnaire. A non-parametric Wilcoxon test was used to assess changes in faecal flora after treatment, repeated-measures ANOVA for the effect of treatment on immune variables and Monte Carlo exact test for differences in symptom improvement.

The improvement in intestinal motility was significantly more reported in the $\mathrm{S}$ group than in the $\mathrm{C}$ group $(P<0.003)$. Also, the cumulative self-reported improvement in any symptom was significantly more frequent in the $\mathrm{S}$ group than in the $\mathrm{C}$ group $(P<0.001)$, although asymptomatic volunteers were significantly more frequent in the $\mathrm{S}$ group in the baseline evaluation $(P<0.01)$. No significant interaction of time $\times$ group was observed for any immunological variable except sL-selectin, which showed a significant decrease in the $\mathrm{S}$ group (see Table). When correlations were analysed in the groups defined by treatment, it was observed that in the $\mathrm{S}$ group baseline $\mathrm{L}-$ selectin levels were correlated with final sICAM-1 levels $(r 0.468 ; P=0.050)$ and baseline sICAM-1 levels were negatively correlated with final L-selectin concentration $(r-0.457 ; P=0.056)$. In contrast, no significant correlations were observed between sL-selectin and sICAM-1 in the $\mathrm{C}$ group. The microbiological analysis of faecal samples showed no differences between the $\mathrm{C}$ and $\mathrm{S}$ groups, probably because a low bacterial load was administered.

\begin{tabular}{|c|c|c|c|c|c|c|c|c|c|}
\hline & \multicolumn{4}{|c|}{ C group (n 18) } & \multicolumn{4}{|c|}{ S group (n 18) } & \multirow[b]{3}{*}{$P$ (ANOVA } \\
\hline & \multicolumn{2}{|c|}{ T1 } & \multicolumn{2}{|c|}{$\mathrm{T} 2$} & \multicolumn{2}{|c|}{$\mathrm{T} 1$} & \multicolumn{2}{|c|}{$\mathrm{T} 2$} & \\
\hline & Mean & $\overline{\mathrm{SD}}$ & Mean & $\overline{\mathrm{SD}}$ & Mean & $\overline{\mathrm{SD}}$ & Mean & $\overline{\mathrm{SD}}$ & \\
\hline $\mathrm{CA}$ & 469 & 85 & 433 & 82 & 434 & 78 & 406 & 50 & 0.674 \\
\hline sVCAM-1 (ng/ml) & 615 & 212 & 575 & 198 & 545 & 126 & 521 & 148 & 0.732 \\
\hline sL-selectin $(\mathrm{ng} / \mathrm{ml})$ & 2586 & 555 & 2560 & 626 & 2724 & 600 & $2389^{*}$ & 420 & 0.050 \\
\hline
\end{tabular}

T1, baseline; T2, after 6 weeks of treatment. Mean value was significantly different from that for T1 (Student's test):
$* P<0.05$.

The present study shows that the daily intake of the synbiotic supplement can have beneficial effects on the intestinal motility of healthy subjects and might improve the serum levels of L-selectin, possibly leading to a more beneficial profile of the inflammatory markers in relation to the prevention of atherosclerosis and CVD.

This work has been supported by LABORATORIOS PHERGAL SA. 